\title{
Who should look after asthma?
}

Most articles on asthma management focus on drug treatment. Broader issues - such as the way patients manage their asthma - are equally important although less frequently subjected to controlled trials. This article addresses some of the non-pharmacological aspects of asthma management. Our discussion is limited to adults with asthma within the National Health Service in the United Kingdom.

When measured against improved asthma control, most of the properly controlled trials of non-pharmacological interventions in the management of asthma have had an unimpressive outcome. ${ }^{1-4}$ This, we believe, is due to two main factors. Firstly, most interventions are not tailored to the individual patient ${ }^{5}$ - for example, the same intervention such as an educational programme is usually given to each patient in a study, irrespective of the severity of the patient's asthma, their educational attainment, and their interest in and understanding of their disease. Secondly, the outcome of such trials is difficult to evaluate; many interventions (such as an educational booklet) are likely to affect an outcome measure (such as hospital admission) in only a relatively small proportion of patients. Even in a large study in which the educational booklet was personalised, many aspects of asthma control were unaffected although hospital admissions were reduced. ${ }^{6}$ Successful asthma care arises from a large number of components, most of which individually have a relatively small effect. Trials which involve a small number of patients and which are conducted over a short period of time are unlikely to have sufficient power to detect a positive effect.

Despite the rather negative results of such trials, most clinicians continue to pursue and most authorities to recommend strategies designed to increase patient understanding and involvement in their management. We share this aim and although, like most doctors, our opinion is influenced by the results of trials, it draws heavily on clinical experience.

Any discussion of asthma management must begin by recognising an enormous variation in the severity of the condition. There are patients who notice occasional chest tightness only during viral infections and those with severe intractable breathlessness; some are well most of the time but experience occasional severe attacks, others have persistently poor lung function; some patients are able to detect deterioration in their asthma, others are not; some patients are disabled by asthma, others are successful in leading normal lives. The advice we offer should reflect the needs of all these groups of patients and clearly needs to be individually tailored.

\section{Which qualities characterise good management?}

\section{AT THE TIME OF DIAGNOSIS}

Asthma may occur for the first time in an adult or may recur in patients who had symptoms as a child. The style in which discussion and management advice is offered to patients at the time of diagnosis may well set the tone for the patient's subsequent attitude to the condition.

At first contact patients can be presented with a great deal of information. It is clearly relevant to explain the nature of the disease and the rationale for treatment and to explore a patient's preconceptions and how it may affect their work and activities. Patients also need to understand the importance of non-drug management - maintaining optimum weight, keeping fit and avoiding cigarette smoke and allergens when possible. However, not only is sharing this information a considerable undertaking, but presenting too much information to patients on one occasion may be counterproductive, particularly if this is done at the time of diagnosis or during a severe attack when they may be preoccupied and doubtful. For most patients a gradual introduction to this information is more sensible. A decision as to who should provide this information will vary according to local resources and expertise; it is more important to ensure that patients have access to information and answers to their questions in a calm and unhurried environment.

\section{ACUTE ASTHMA ATTACKS}

Most acute attacks of asthma are seen by general practitioners and most patients do not come to hospital. Good management at this time requires a doctor who is competent, up to date, and able to give appropriate treatment quickly and safely. Medical attention both in and outside hospital must be readily accessible. General practitioners should be able to assess severity and act accordingly. Once the acute attack has responded to treatment the patient and doctor and/or nurse need to consider the factors preceding the acute attack and try to determine whether, with a different approach, it could have been prevented.

\section{CONTINUING FOLLOW UP}

The extent and nature of follow up of asthma depends on the severity of the condition. Patients with stable mild disease (BTS Guidelines steps 1 or 2) may prefer minimal contact with doctors. In many instances this is a reasonable choice which should not be discouraged in patients with near normal lung function, unrestricted activities, good inhaler technique, and sensible use of medication. Patients are unlikely to take advice if their experience is that contact with the health services is more trouble than their asthma. ${ }^{7}$ For these patients an infrequent or opportunistic review may be acceptable. However, many patients require more frequent follow up, either because they feel less confident of their asthma or because it is more difficult to control.

Good management requires competence and consistency, especially when patients are being seen by several doctors - a consideration which applies both to general practice and hospital care. It would seem sensible for agreed treatment schedules and shared care plans to be developed, but in practice this has been slow to happen. It sounds easy and sensible for patients to have a "cooperation" card or action plan but there is less certainty about what information it should contain. Is the card primarily for the patient or the doctor? Patients are unlikely to use a card (or remember to bring it to the surgery or hospital) unless they find it helpful, or if their efforts to collect data are ignored at review. Too much information on a card could be counterproductive. It is unlikely that a single card or plan will cover the needs of all patients, and we need to develop and assess a range of cards to cover variations in asthma severity and the information needed by individual patients. 


\section{When should patients under follow up be referred to a respiratory physician?}

The last decade or so has seen a welcome increase in the ability and enthusiasm to manage asthma in primary care, although obviously there is a considerable variation between individual general practitioners. Whether patients with late onset asthma should be referred to a consultant respiratory physician will therefore depend on a number of factors including the disease, the patient, the general practitioner, and local circumstances.

Some patients who develop late onset asthma may wish to have a second opinion, and this is not unreasonable. Patients with a recrudescence of childhood asthma may be less likely to require or seek referral, although the decision to refer usually reflects the confidence of the patient and the general practitioner as much as the severity of the disease. Further outpatient investigations may confirm or occasionally refute the diagnosis, and some of the causes underlying late onset asthma such as aspirin sensitivity and occupation may be unearthed.

Referral to a respiratory physician should be considered under the following circumstances: (1) where there are doubts as to whether the patient has asthma; (2) failure to respond to treatment or the need for large or increasing doses of medication; (3) a large disparity between symptoms, objective evidence of airways obstruction and use of treatment; (4) any suspicion of occupational asthma; (5) where contributing factors such as gastro-oesophageal reflux, or drug or food intolerance are suspected.

Overuse of treatment is a problem in a few patients. Overuse of $\beta$ agonists is usually due to inadequate control of underlying inflammation, although some patients are perhaps overdependent on their $\beta$ agonist inhaler. Regular recording of peak flow rates should clarify whether control is satisfactory. Overuse of oral prednisolone will be rare for an individual general practitioner but is seen in specialist practice and appears to be more common in young women. Some of these patients have inappropriate hyperventilation in addition to their asthma, and both the patient and doctor may have difficulty in distinguishing between the two. General concerns about undertreatment of acute asthma means that some of these patients are given rescue courses of prednisolone unnecessarily frequently so that they become Cushingoid. It is unusual for patients with chronic persistent asthma to require more than $7.5 \mathrm{mg}$ prednisolone/day and patients who require more should be assessed by a respiratory physician. Such patients can be very difficult to manage and a joint approach is usually helpful.

\section{Patient self-management}

Perhaps the major change in the management of asthma over the last decade has been the shift of emphasis towards educating patients to look after their asthma themselves. This move to decrease dependency and encourage guided self-management is clearly appropriate, and the process has been helped by the increased availability in hospital and in general practice of nurses with training in asthma care. This nursing input is complementary to the medical input and has been a welcome additional resource. It enables patients to receive additional information and often a greater opportunity to discuss their concerns - two very important facets of good management.

\section{Where should the emphasis be in the future?}

We strongly believe that patient education is the cornerstone of better asthma management. The two areas where improvements are most needed in the foreseeable future are in improving patient education and in improving communication and liaison between primary and secondary care. Some specific measures which might help are as follows:

1 Nurse training. Despite the excellent contribution of -1 several training centres, not all nurses looking after 음 patients with asthma have sufficient knowledge and $\stackrel{x}{x}$ expertise to undertake this work properly. Training courses need to be more widely available, and asthma $\stackrel{9}{\rightarrow}$ nurses in hospital and general practice would be helped by strong local networks. Individual strategies such as $\frac{\bar{\sigma}}{\bar{\phi}}$ nurse drop-in clinics and nurse-run classes for newly $\frac{\widehat{D}}{\phi}$ diagnosed asthma need to be tried and evaluated.

2 Doctor education. The extent to which general prac- $ळ$ titioners and hospital doctors are managing asthma $\overrightarrow{0}$ adequately is uncertain and probably varies in different $\overrightarrow{\vec{H}}$ parts of the country. As in all areas of medicine, continuing education tends to reach the converted and $\vec{\overrightarrow{ }}$ ways in which audit might help need to be assessed. Management in hospital is likely to be improved by use of local protocols and by rotating junior doctors if through respiratory wards.

3 Admissions policy in acute asthma attacks. During an 은 individual attack admission under a respiratory phys- $\overrightarrow{-}$ ician is likely to be in the patient's interest. ${ }^{8}$ In the long term the benefits are less clear. Such specialisation $\underset{\nabla}{\Phi}$ means that some junior doctors and medical students $\vec{\theta}$ will have little experience in the assessment and man- of agement of severe asthma and may then feel less confident in looking after such patients in general practice. 우 Furthermore, given the prevalence of asthma, a considerable increase in the number of chest physicians would be required. For the foreseeable future, at least, $\frac{\square}{\triangleright}$ a reasonable target would be for all patients admitted $\underset{F}{\vec{F}}$ to hospital with an acute exacerbation of asthma to be seen once during their inpatient stay by a respiratory $\vec{\partial}$ nurse or a respiratory physician (consultant or registrar). With increased experience and training respiratory nurses would be able to identify patients at particular risk and those who would benefit from more $\underset{x}{\tilde{x}}$ specialist input.

4 Improving communications between hospital and general practice. The benefits of good management during an acute attack of asthma in hospital are lost if the patient, general practitioner, and practice nurse are not involved $\stackrel{ }{3}$ in - or at the very least informed of - subsequent $\frac{D}{0}$ management plans and arrangements for follow up. How might this be improved? Details of management $N$ should be given to the patient on discharge and to $N$ the general practitioner soon thereafter. With shorter N inpatient stays there is an increased responsibility on 0 hospitals to share information on discharge by making

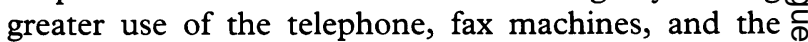
nurse network. Discussion of shared protocols at district $\stackrel{?}{+}$ level should involve such procedures. More work is needed to develop patient-based cards and action plans that are acceptable to patients, specialists, and general practitioners.

The needs of a patient with asthma include easy access to advice and preventative treatment when well, and rapid access to medical help for exacerbations and to specialist attention and support when indicated. Providing an optimum level of care for the large number of patients with asthma, when severity varies widely and sometimes unpredictably, is a major challenge. A great deal of progress has been made over the last 10 years by hospital physicians, general practitioners, and nurses with the support of various bodies including the National Asthma Campaign. Efforts 
need to continue, however, as many patients are still not receiving optimum care. How this care should be organised will depend on local resources and should be discussed at district level by all concerned. An integrated programme worked well in the Scottish Highlands for patients with moderately severe asthma and is one of several models that could and should be tried elsewhere. ${ }^{9}$

Division of Respiratory Medicine,

A E TATTERSFIELD

City Hospital,

Nottingham NG5 $1 P B$

UK

Sherrington Park Medical Practice,

402 Mansfield Road,

Nottingham NG5 2Ef,

$U K$
1 Jenkinson D, Davison J, Jones S, Hawtin P. Comparison of effects of a self management booklet and audio cassette for patients with asthma. $B M \mathcal{F}$ 1988;297:267-70.

2 Hill R, Williams J, Britton J, Tattersfield A. Can morbidity associated with untreated asthma in primary school children be reduced? A controlled intervention study. BMF 1991;303:1169-74.

3 Charlton I, Charlton G, Broomfield J, Mullee MA. Evaluation of peak flow and symptoms only self management plans for control of asthma in general practice. $B M 7$ 1990;301:1355-9.

4 Drummond N, Abdalla M, Beattie JAG, Buckingham JK, Lindsay T, Osman $\mathrm{L}$, et al. Effectiveness of routine self monitoring of peak flow in patients with asthma. BMF 1994;308:564-7.

5 Brewis RAL. Patient education, self-management plans and peak flow measurement. Respir Med 1991;85:457-62.

6 Osman LM, Abdalla MI, Beattie JAG, Ross SJ, Russell IT, Friend JA, et al. Reducing hospital admission through computer supported education for asthma patients. $B M 7$ 1994;308:568-71.

7 Keeley D. How to achieve better outcome in treatment of asthma in general practice. BMf 1993;307:1261-3.

W HOLMES 8 Bucknall CE, Robertson C, Moran F, Stevenson RD. Differences in hospital asthma management. Lancet 1988;i:748-50.

9 Drummond N, Abdalla M, Buckingham JK, Beattie JAG, Lindsay T, Osman L, et al. Integrated care for asthma: a clinical, social, and economic evaluation. BMF 1994;308:559-64. 